\title{
Article
}

\section{Two Forms of An Inverse Operator to the Generalized Bessel Potential}

\author{
Akhmed Dzhabrailov ${ }^{1}$ (D), Yuri Luchko ${ }^{2, *}$ and Elina Shishkina ${ }^{3,4}$ (D) \\ 1 Department of Mathematical Analysis, Algebra and Geometry, Chechen State University, Sheripov Str. 32, \\ 364024 Grozny, Russia; ahmed_0065@mail.ru \\ 2 Department of Mathematics, Physics, and Chemistry, Beuth Technical University of Applied Sciences Berlin, \\ Luxemburger Str. 10, 13353 Berlin, Germany \\ 3 Department of Mathematical and Applied Analysis, Voronezh State University, Universitetskaya pl., 1, \\ 394018 Voronezh, Russia; ilina_dico@mail.ru \\ 4 Department of Applied Mathematics and Computer Modeling, Belgorod State National Research University \\ (BelGU), Pobedy Street, 85, 308015 Belgorod, Russia \\ * Correspondence: luchko@beuth-hochschule.de
}

check for updates

Citation: Dzhabrailov A.; Luchko, Y.; Shishkina, E. Two Forms of An Inverse Operator to the Generalized Bessel Potential. Axioms 2021, 10, 232. https://doi.org/10.3390/ axioms10030232

Academic Editor: Palle E. T. Jorgensen

Received: 28 July 2021

Accepted: 14 September 2021

Published: 18 September 2021

Publisher's Note: MDPI stays neutral with regard to jurisdictional claims in published maps and institutional affiliations.

Copyright: (c) 2021 by the authors. Licensee MDPI, Basel, Switzerland. This article is an open access article distributed under the terms and conditions of the Creative Commons Attribution (CC BY) license (https:/ / creativecommons.org/licenses/by/ $4.0 /)$.

\begin{abstract}
In this paper, we treat a convolution-type operator called the generalized Bessel potential. Our main result is the derivation of two different forms of its inversion. The first inversion is provided in terms of an approximative inverse operator using the method of an improving multiplier. The second one employs the regularization technique for the divergent integrals in the form of the appropriate segments of the Taylor-Delsarte series.
\end{abstract}

Keywords: generalized Bessel potential; inverse operator; approximative inverse operator; Hadamard regularisation

MSC: 31B15; 47G10; 44A15; 46E30

\section{Introduction}

The potential theory originates from the theory of electrostatic and gravitational potentials and the Laplace, wave, Helmholtz, and Poisson equations. The famous Riesz potentials are known to be the realizations of the real negative powers of the Laplace and wave operators. In the meantime, a lot of attention in the potential theory is given to the Bessel potential

$$
\mathbf{G}^{\alpha} f(x)=\int_{\mathbb{R}^{n}} \mathcal{G}_{\alpha}(x-y) f(y) d y, \quad \alpha>0,
$$

where

$$
\mathcal{G}_{\alpha}(x)=\frac{2^{\frac{2-n-\alpha}{2}}}{\pi^{\frac{n}{2}} \Gamma\left(\frac{\alpha}{2}\right)} \frac{K_{\frac{n-\alpha}{2}}(|x|)}{|x|^{\frac{n-\alpha}{2}}}
$$

and $K_{v}$ stands for the modified Bessel functions of the second kind. The operator $\mathrm{G}^{\alpha}$ can be interpreted as a realization of the real negative powers of the operator $(I-\Delta)$. In particular, the Bessel potential was treated by N. Aronzajn and K.T. Smith in [1] and by Calderon in [2]. It is worth mentioning that the Bessel potentials generate some special spaces of functions with a fractional smoothness $\alpha$. They are very useful for the investigation of some fractional PDEs of the elliptic type. These spaces are an extension of the Sobolev spaces $L_{p}^{m}\left(\mathbb{R}^{n}\right)$ to the case of the fractional order $\alpha$. They are often referred to as Sobolev spaces of fractional order. The first results regarding the spaces of Bessel potentials were obtained by I. Stein in [3] in the case $0<\alpha<2$ and by Lizorkin in [4] in the general case. The inversion of the Bessel potentials was first derived by V.A. Nogin in $[5,6]$ using the hypersingular integrals. Later on, V.S. Guliev and Z.V. Safarov studied the Bessel potentials generated by the Bessel differential operators in [7]. In particular, they proved the boundedness of these 
potentials in the weighted Lebesgue spaces and derived some embedding theorems in $B_{k, n}$-Sobolev-Liouville spaces. In [8], the Young inequality for the B-convolution operators in the B-Bessel potential spaces was proved, and the Bessel potentials were characterized in terms of the B-Lizorkin-Triebel spaces. The optimal embedding of spaces of Bessel-type potentials were obtained in [9-11].

An inversion formula for the Bessel potential $\mathrm{G}^{\alpha}$ was derived in [12] using a special type of the weighted wavelet transforms. Further investigation of the hypersingular integrals associated to $\mathrm{G}^{\alpha}$ was performed in [13]. In [14], the fractional powers $\left(I-\left(\sum_{k=1}^{n} \frac{\partial^{2}}{\partial x_{k}^{2}}+\frac{2 v}{x_{n}} \frac{\partial}{\partial x_{k}}\right)\right)^{-\alpha}$ of the operator $\left(I-\left(\sum_{k=1}^{n} \frac{\partial^{2}}{\partial x_{k}^{2}}+\frac{2 v}{x_{n}} \frac{\partial}{\partial x_{k}}\right)\right), v>0$ were introduced, and their approximation properties were studied as $\alpha$ tends to $0+$.

In this paper, we first construct the positive fractional powers of the operator $\left(I-\Delta_{\gamma}\right)$, where $\Delta_{\gamma}=\sum_{k=1}^{n}\left(B_{\gamma_{k}}\right)_{x_{k}}$ is the Laplace-Bessel operator with $\left(B_{\gamma_{k}}\right)_{x_{k}}=\frac{\partial^{2}}{\partial x_{k}^{2}}+\frac{\gamma_{k}}{x_{k}} \frac{\partial}{\partial x_{k}}$. The negative fractional powers of this operator are the generalized Bessel potentials considered in [15]. The most important difference between the generalized Riesz potential $\left(-\Delta_{\gamma}\right)^{-\frac{\alpha}{2}}$, $\alpha>0$, and the generalized Bessel potential $\left(I-\Delta_{\gamma}\right)^{-\frac{\alpha}{2}}$ is that the kernel of the generalized Bessel potential has an exponential decay at infinity.

It is worth mentioning that the integral equations involving the Bessel potentials and their generalizations are actively employed, say, for the investigation of the corresponding PDEs, such as the static Shrödinger equations with the critical and the super-critical exponents.

The generalized Bessel potential or Bessel B-potential is defined by the formula [15]

$$
\left(\mathbf{G}_{\gamma}^{\alpha} \varphi\right)(x)=\left(G_{\alpha}^{\gamma} * \varphi\right)_{\gamma}=\int_{\mathbb{R}_{+}^{n}} G_{\alpha}^{\gamma}(y)\left({ }^{\gamma} \mathbf{T}_{x}^{y} \varphi(x)\right) y^{\gamma} d y, \quad \alpha>0
$$

with the kernel

$$
G_{\alpha}^{\gamma}(x)=\frac{2^{\frac{n-|\gamma|-\alpha}{2}+1}}{|x|^{\frac{n+|\gamma|-\alpha}{2}} \Gamma\left(\frac{\alpha}{2}\right) \prod_{i=1}^{n} \Gamma\left(\frac{\gamma_{i}+1}{2}\right)} K_{\frac{n+|\gamma|-\alpha}{2}}(|x|)
$$

and the generalized translation ${ }^{\gamma} \mathbf{T}_{x}^{y}$ defined by the Formula (17) (see the next section). Equation (1) realizes the negative fractional power $\left(I-\Delta_{\gamma}\right)^{-\alpha / 2}, \alpha>0$, where $I$ is the identity operator, and $\Delta_{\gamma}$ is the Laplace-Bessel operator

$$
\Delta_{\gamma}=\left(\Delta_{\gamma}\right)_{x}=\sum_{k=1}^{n}\left(B_{\gamma_{k}}\right)_{x_{k}}
$$

and

$$
\left(B_{\gamma}\right)_{t}=\frac{\partial^{2}}{\partial t^{2}}+\frac{\gamma}{t} \frac{\partial}{\partial t}=\frac{1}{t \gamma} \frac{\partial}{\partial t} t^{\gamma} \frac{\partial}{\partial t^{\prime}}, \quad t>0, \quad \gamma \in \mathbb{R}
$$

is the Bessel operator. The results derived in [15] allow us to represent the generalized Bessel potential in the form

$$
\mathbf{G}_{\gamma}^{\alpha} \varphi=\mathbf{F}_{\gamma}^{-1}\left(1+|x|^{2}\right)^{-\frac{\alpha}{2}} \mathbf{F}_{\gamma} \varphi, \quad \alpha>0,
$$

where $\mathbf{F}_{\gamma}$ stands for the multidimensional Hankel transform defined by the Equation (23) (see the next section).

In this paper, we construct two different explicit forms of the operator inverse to Equation (1) in the weighted Lebesgue spaces, or, in other words, we get two representations of the positive fractional powers $\left(I-\Delta_{\gamma}\right)^{\alpha / 2}, \alpha>0$. The operator $\left(I-\Delta_{\gamma}\right)^{\alpha / 2}, \alpha>0$ is first constructed by the approximative inverse operator (AIO) method, and then, another realization is obtained using the so-called B-hypersingular integral with a stabilizing characteristic. 


\section{Preliminary Results}

In what follows, by $\mathbb{R}_{+}^{n}$, we denote the open orthant

$$
\mathbb{R}_{+}^{n}=\left\{x=\left(x_{1}, \ldots, x_{n}\right) \in \mathbb{R}^{n}, x_{1}>0, \ldots, x_{n}>0\right\} .
$$

and by $\overline{\mathbb{R}}_{+}^{n}$ its closure:

$$
\overline{\mathbb{R}}_{+}^{n}=\left\{x=\left(x_{1}, \ldots, x_{n}\right) \in \mathbb{R}^{n}, x_{1} \geq 0, \ldots, x_{n} \geq 0\right\} .
$$

By $\gamma=\left(\gamma_{1}, \ldots, \gamma_{n}\right)$, we denote a multi-index that consists of some positive real numbers $\gamma_{i}$, $i=1, \ldots, n$, and $|\gamma|=\gamma_{1}+\ldots+\gamma_{n}$.

A part of a sphere of radius $r$ with the center at the origin that belongs to $\mathbb{R}_{+}^{n}$ will be denoted by $S_{r}^{+}(n)$ :

$$
S_{r}^{+}(n)=\left\{x \in \overline{\mathbb{R}}_{+}^{n}:|x|=r\right\} \cup\left\{x \in \overline{\mathbb{R}}_{+}^{n}: x_{i}=0,|x| \leq r, i=1, \ldots, n\right\} .
$$

For our derivations, we employ the following two formulas (see, e.g., the formulas 1.107 and 3.140 in [16]):

$$
\begin{gathered}
\int_{S_{1}^{+}(n)} \sigma^{\gamma} d S=\frac{\prod_{i=1}^{n} \Gamma\left(\frac{\gamma_{i}+1}{2}\right)}{2^{n-1} \Gamma\left(\frac{n+|\gamma|}{2}\right)}, \quad \sigma^{\gamma}=\prod_{i=1}^{n} \sigma_{i}^{\gamma_{i}}, \\
\int_{S_{1}^{+}(n)} \mathbf{j}_{\gamma}(x, r \sigma) \sigma^{\gamma} d S=\frac{\prod_{i=1}^{n} \Gamma\left(\frac{\gamma_{i}+1}{2}\right)}{2^{n-1} \Gamma\left(\frac{n+|\gamma|}{2}\right)} j_{\frac{n+|\gamma|}{2}-1}(r|x|),
\end{gathered}
$$

where

$$
\mathbf{j}_{\gamma}(x ; \xi)=\prod_{i=1}^{n} j_{\frac{\gamma_{i}-1}{2}}\left(x_{i} \xi_{i}\right)
$$

and $j_{v}$ stands for the normalized Bessel function of the first kind

$$
j_{v}(x)=\frac{2^{v} \Gamma(v+1)}{x^{v}} J_{v}(x)
$$

where $J_{v}$ is the Bessel function of the first kind.

Let $\Omega$ be finite or infinite open set in $\mathbb{R}^{n}$ symmetric with respect to each hyperplane $x_{i}=0, i=1, \ldots, n, \Omega_{+}=\Omega \cap \mathbb{R}_{+}^{n}$, and $\bar{\Omega}_{+}=\Omega \cap \overline{\mathbb{R}}_{+}^{n}$, where $\overline{\mathbb{R}}_{+}^{n}=\left\{x=\left(x_{1}, \ldots, x_{n}\right) \in \mathbb{R}^{n}\right.$, $\left.x_{1} \geq 0, \ldots, x_{n} \geq 0\right\}$. In this paper, we employ the class $C^{m}\left(\Omega_{+}\right)$of functions that are $m-$ times differentiable on $\Omega_{+}$. By $C^{m}\left(\bar{\Omega}_{+}\right)$, we denote the subset of $C^{m}\left(\Omega_{+}\right)$that contains the function with all continuous derivatives with respect to $x_{i}$ for any $i=1, \ldots, n$ up to $x_{i}=0$. The class $C_{e v}^{m}\left(\bar{\Omega}_{+}\right)$of functions consists of all functions from $C^{m}\left(\bar{\Omega}_{+}\right)$such that $\left.\frac{\partial^{2 k+1} f}{\partial x_{i}^{2 k+1}}\right|_{x_{i}=0}=0$ for all non-negative integers $k \leq \frac{m-1}{2}$ (see [17], p. 21). In what follows, we denote the space $C_{e v}^{m}\left(\overline{\mathbb{R}}_{+}^{n}\right)$ just by $C_{e v}^{m}$ and set

$$
C_{e v}^{\infty}\left(\bar{\Omega}_{+}\right)=\bigcap C_{e v}^{m}\left(\bar{\Omega}_{+}\right),
$$

where the intersection is taken over all $m \in \mathbb{N}_{0}$ and all $C_{e v}^{\infty}\left(\overline{\mathbb{R}}_{+}\right)=C_{e v}^{\infty}$.

Our results in the next sections will be formulated for the functions from some spaces of generalized functions. Now we proceed with a description of these spaces. As a space 
of the basic (or test) functions, the following subspace of the space of rapidly decreasing functions will be used:

$$
S_{e v}=\left\{f \in C_{e v}^{\infty}: \sup _{x \in \mathbb{R}_{+}^{n}}\left|x^{\alpha} D^{\beta} f(x)\right|<\infty \quad \forall \alpha, \beta \in \mathbb{Z}_{+}^{n}\right\},
$$

where $\alpha=\left(\alpha_{1}, \ldots, \alpha_{n}\right), \beta=\left(\beta_{1}, \ldots, \beta_{n}\right), \alpha_{1}, \ldots, \alpha_{n}, \beta_{1}, \ldots, \beta_{n}$ are integer non-negative numbers, $x^{\alpha}=x_{1}^{\alpha_{1}} x_{2}^{\alpha_{2}} \ldots x_{n}^{\alpha_{n}}, D^{\beta}=D_{x_{1}}^{\beta_{1}} \ldots D_{x_{n}}^{\beta_{n}}, D_{x_{j}}=\frac{\partial}{\partial x_{j}}$.

As usual, the space of the weighted generalized functions $S_{e v}^{\prime}\left(\mathbb{R}_{+}^{n}\right)=S_{e v}^{\prime}$ is defined as a class of continuous linear functionals that map the test functions from $f \in S_{e v}$ into the set of real numbers. Each function $u$ such that $u(x) x^{\gamma} \in L_{1, l o c}, x^{\gamma}=x_{1}^{\gamma_{1}} \ldots x_{n}^{\gamma_{n}}$ will be identified with the functional $u \in S_{e v}^{\prime}\left(\mathbb{R}_{+}^{n}\right)=S_{e v}^{\prime}$ that acts according to the formula

$$
(u, f)_{\gamma}=\int_{\mathbb{R}_{+}^{n}} u(x) f(x) x^{\gamma} d x, \quad f \in S_{e v} .
$$

The generalized functions $u \in S_{e v}^{\prime}$ defined by Equation (11) with $u(x) x^{\gamma} \in L_{1, l o c}$ will be called regular weighted generalized functions. All other generalized functions $u \in S_{e v}^{\prime}$ will be called singular weighted generalized functions.

By $L_{p}^{\gamma}\left(\mathbb{R}_{+}^{n}\right)=L_{p}^{\gamma}, 1 \leq p<\infty$, we denote the space of all functions measurable in $\mathbb{R}_{+}^{n}$ even with respect to each variable $x_{i}, i=1, \ldots, n$ such that

$$
\int_{\mathbb{R}_{+}^{n}}|f(x)|^{p} x^{\gamma} d x<\infty
$$

where $x^{\gamma}=\prod_{i=1}^{n} x_{i}^{\gamma_{i}}$. For a real number $p \geq 1$, the $L_{p}^{\gamma}$-norm of $f$ is defined by

$$
\|f\|_{L_{p}^{\gamma}\left(\mathbb{R}_{+}^{n}\right)}=\|f\|_{p, \gamma}=\left(\int_{\mathbb{R}_{+}^{n}}|f(x)|^{p} x^{\gamma} d x\right)^{1 / p} .
$$

It is known (see [17]) that $L_{p}^{\gamma}$ is a Banach space.

The modified Bessel functions $I_{\alpha}(x)$ and $K_{\alpha}(x)$ (occasionally called the hyperbolic Bessel functions) of the first and second kind, respectively, are defined as follows (see [18,19]):

$$
\begin{aligned}
I_{\alpha}(x)=i^{-\alpha} J_{\alpha}(i x) & =\sum_{m=0}^{\infty} \frac{1}{m ! \Gamma(m+\alpha+1)}\left(\frac{x}{2}\right)^{2 m+\alpha}, \\
K_{\alpha}(x) & =\frac{\pi}{2} \frac{I_{-\alpha}(x)-I_{\alpha}(x)}{\sin (\alpha \pi)},
\end{aligned}
$$

where $\alpha$ is non-integer. For integer values of the parameter $\alpha$, the functions are defined by taking the limit in the expressions presented above. Obviously, the function $K_{\alpha}(x)$ is an even function, i.e., the relation

$$
K_{\alpha}(x)=K_{-\alpha}(x)
$$

holds valid. For $r \rightarrow 0(0<|r| \ll \sqrt{v+1})$, the known asymptotic formula for the function $K_{\alpha}(x)$ is as follows [20]:

$$
K_{v}(r) \sim \begin{cases}-\ln \left(\frac{r}{2}\right)-\vartheta, & \text { if } v=0 \\ \frac{\Gamma(v)}{2^{1-v}} r^{-v}, & \text { if } v>0\end{cases}
$$


where

$$
\vartheta=\lim _{n \rightarrow \infty}\left(-\ln n+\sum_{k=1}^{n} \frac{1}{k}\right)=\int_{1}^{\infty}\left(-\frac{1}{x}+\frac{1}{\lfloor x\rfloor}\right) d x
$$

is the Euler-Mascheroni constant.

For $r \rightarrow+\infty$, we have another known asymptotic formula:

$$
K_{v}(r) \sim \sqrt{\frac{\pi}{2 r}} e^{-r}
$$

Some of our results will be formulated in terms of the generalized translation defined as follows (see [21]):

$$
\begin{gathered}
\gamma T_{x}^{y} f(x)=C(\gamma) \int_{0}^{\pi} f\left(\sqrt{x^{2}+y^{2}-2 x y \cos \varphi}\right) \sin ^{\gamma-1} \varphi d \varphi, \quad \gamma>0, \\
C(\gamma)=\left(\int_{0}^{\pi} \sin ^{\gamma-1} \varphi d \varphi\right)^{-1}=\frac{\Gamma\left(\frac{\gamma+1}{2}\right)}{\sqrt{\pi} \Gamma\left(\frac{\gamma}{2}\right)}, \quad \gamma>0 .
\end{gathered}
$$

For $\gamma=0$, the generalized translation ${ }^{\gamma} T_{x}^{y}$ is reduced to the following simple translation:

$$
{ }^{0} T_{x}^{y}=T_{x}^{y} f(x)=\frac{f(x+y)+f(x-y)}{2} .
$$

The multidimensional generalized translation is defined by the equality

$$
\left({ }^{\gamma} \mathbf{T}_{x}^{y} f\right)(x)={ }^{\gamma} \mathbf{T}_{x}^{y} f(x)=\left({ }^{\gamma_{1}} T_{x_{1} \ldots}^{y_{1} \ldots}{ }^{\gamma_{n}} T_{x_{n}}^{y_{n}} f\right)(x),
$$

where each of the one-dimensional generalized translations $\gamma_{i} T_{x_{i}}^{y_{i}}, i=1, \ldots, n$ acts according to Equation (17) for $\gamma>0$ and according to Equation (18) for $\gamma=0$.

Another object useful for our derivations is a generalized convolution generated by a multidimensional generalized translation ${ }^{\gamma} \mathbf{T}_{x}^{y}$. It is defined as follows:

$$
(f * g)_{\gamma}(x)=(f * g)_{\gamma}=\int_{\mathbb{R}_{+}^{n}} f(y)\left({ }^{\gamma} \mathbf{T}_{x g}^{y} g\right)(x) y^{\gamma} d y .
$$

It is worth mentioning that the operators ${ }^{\gamma} T_{x}^{h}$ can be expanded in terms of the powers of the Bessel operator by the following formula from [21] (compare to the well-known representation of the shift operator in terms of powers of the differentiation operator $D$ ):

$$
{ }^{\gamma} T_{x}^{h} u(x)=\sum_{k=0}^{m} \varphi_{k}(h) B_{\gamma}^{k} u(x)+r_{m}(x, h),
$$

where

$$
\begin{gathered}
\varphi_{k}(h)=\frac{1}{k !} \frac{\Gamma\left(\frac{\gamma+1}{2}\right)}{\Gamma\left(\frac{\gamma+1}{2}+k\right)}\left(\frac{h}{2}\right)^{2 k}, \\
r_{m}(x, h)=\left.\varphi_{m+1}(h) B_{\gamma}^{m+1} u(\xi)\right|_{\xi=x+\theta h}, \quad-1<\theta<1 .
\end{gathered}
$$

In particular, we obtain

$$
\varphi_{0}(y)=1, \quad \varphi_{1}(h)=\frac{\Gamma\left(\frac{\gamma+1}{2}\right)}{\Gamma\left(\frac{\gamma+1}{2}+1\right)}\left(\frac{h}{2}\right)^{2}=\frac{h^{2}}{2(\gamma+1)} .
$$


Equation (21) is called the Taylor-Delsarte formula. Letting $m$ go to infinity in Equation (21), we arrive at the the Taylor-Delsarte series

$$
{ }^{\gamma} T_{x}^{h} u(x)=\sum_{k=0}^{\infty} \varphi_{k}(h) B_{\gamma}^{k} u(x)
$$

The Taylor-Delsarte Formula (21) can be easily generalized to the case of functions depending on several variables. In what follows, by

$$
\left(P_{y}^{l-1} f\right)(x)=\sum_{|2 m| \leq l-1} \zeta_{m}^{\gamma}\left(\mathcal{B}_{x}^{m} f\right)(x) y^{2 m}
$$

we denote a segment of the multidimensional Taylor-Delsarte series, where $m=\left(m_{1}, \ldots, m_{n}\right)$ is a multi-index containing the non-negative integers, $y^{2 m}=y_{1}^{2 m_{1}} \ldots y_{n}^{2 m_{n}},\left(\mathcal{B}_{x}^{m} f\right)(x)=$ $B_{\gamma_{1}}^{m_{1}} \ldots B_{\gamma_{n}}^{m_{n}} f\left(x_{1}, \ldots, x_{n}\right), \zeta_{m}^{\gamma}=\frac{1}{2^{2|m|} m !} \prod_{i=1}^{n} \frac{\Gamma\left(\frac{\gamma_{i}+1}{2}\right)}{\Gamma\left(m_{i}+\frac{\gamma_{i}+1}{2}\right)}$, and $m !=m_{1} ! \cdot \ldots \cdot m_{n} !$

In this paper, we also employ the multidimensional Hankel transform with the kernel defined by Equation (9) acting on the functions $f \in L_{1}^{\gamma}\left(\mathbb{R}_{+}^{n}\right)$. It is provided by the formula

$$
\mathbf{F}_{\gamma}[f](\xi)=\mathbf{F}_{\gamma}[f(x)](\xi)=\widehat{f}(\xi)=\int_{\mathbb{R}_{+}^{n}} f(x) \mathbf{j}_{\gamma}(x ; \xi) x^{\gamma} d x
$$

where $\mathbb{R}_{+}^{n}$ is defined by Equation (6), the kernel $\mathbf{j}_{\gamma}$ is given by Equation (9), and $x^{\gamma}=\prod_{i=1}^{n} x_{i}^{\gamma_{i}}$. Let $f \in L_{1}^{\gamma}\left(\mathbb{R}_{+}\right)$be a function of bounded variation in a neighborhood of a point $x$ of its continuity. Then, for $\gamma>0$, the following inversion formula for the multidimensional Hankel transform holds valid:

$$
\mathbf{F}_{\gamma}^{-1}[\widehat{f}(\xi)](x)=f(x)=\frac{2^{n-|\gamma|}}{\prod_{j=1}^{n} \Gamma^{2}\left(\frac{\gamma_{j}+1}{2}\right)} \int_{\mathbb{R}_{+}^{n}} \mathbf{j}_{\gamma}(x, \xi) \widehat{f}(\xi) \xi^{\gamma} d \xi
$$

For the multidimensional Hankel transform and the following convolution operator with respect to the generalized convolution

$$
A f=(T * f)_{\gamma}, \quad f \in S_{e v}
$$

the convolution theorem

$$
\mathbf{F}_{\gamma}[A f]=\mathbf{F}_{\gamma}\left[(T * f)_{\gamma}\right]=\mathbf{F}_{\gamma}[T] \cdot \mathbf{F}_{\gamma}[f]
$$

holds valid (see [16], p. 156, formula 3.176).

\section{Some Useful Properties of the General Poisson Kernel}

For our construction of an inverse operator to the generalized Bessel potential in Equation (1) by the method of approximative inverse operators, we employ the generalized convolution with the general Poisson kernel in the form

$$
P_{\gamma}(x, \varepsilon)=\frac{2^{n} \Gamma\left(\frac{n+|\gamma|+1}{2}\right)}{\sqrt{\pi} \prod_{j=1}^{n} \Gamma\left(\frac{\gamma_{j}+1}{2}\right)} \varepsilon\left(\varepsilon^{2}+|x|^{2}\right)^{-\frac{n+|\gamma|+1}{2}}, \quad \varepsilon>0 .
$$

In this section, we present some properties of this kernel needed for the further discussions and start with the following result: 
Lemma 1. The image of the function $e^{-\varepsilon|x|}, \varepsilon>0$ by the Hankel transform defined by Equation (23) can be represented in the form

$$
\mathbf{F}_{\gamma}\left[e^{-\varepsilon|x|}\right](\xi)=\frac{2^{|\gamma|} \mathcal{\varepsilon} \prod_{i=1}^{n} \Gamma\left(\frac{\gamma_{i}+1}{2}\right) \Gamma\left(\frac{n+|\gamma|+1}{2}\right)}{\sqrt{\pi}\left(\varepsilon^{2}+|\xi|^{2}\right)^{\frac{n+|\gamma|+1}{2}}} .
$$

Proof. By definition and employing a simple variables substitution, we first get the representation

$$
\begin{gathered}
\mathbf{F}_{\gamma}\left[e^{-\varepsilon|x|}\right](\xi)=\int_{\mathbb{R}_{+}^{n}} e^{-\varepsilon|x|} \mathbf{j}_{\gamma}(x ; \xi) x^{\gamma} d x=\{x=\rho \sigma\}= \\
=\int_{0}^{\infty} e^{-\varepsilon \rho} \rho^{n+|\gamma|-1} d \rho \int_{S_{1}^{+}(n)} \mathbf{j}_{\gamma}(\rho \sigma ; \xi) \sigma^{\gamma} d S .
\end{gathered}
$$

Then, applying Equation (8), we obtain

$$
\begin{gathered}
\mathbf{F}_{\gamma}\left[e^{-\varepsilon|x|}\right](\xi)=\frac{\prod_{i=1}^{n} \Gamma\left(\frac{\gamma_{i}+1}{2}\right)}{2^{n-1} \Gamma\left(\frac{n+|\gamma|}{2}\right)} \int_{0}^{\infty} e^{-\varepsilon \rho} j_{\frac{n+|\gamma|}{2}-1}(\rho|\xi|) \rho^{n+|\gamma|-1} d \rho= \\
=\frac{\prod_{i=1}^{n} \Gamma\left(\frac{\gamma_{i}+1}{2}\right)}{2^{\frac{n-|\gamma|}{2}}|\xi|^{\frac{n+|\gamma|}{2}}-1} \int_{0}^{\infty} e^{-\varepsilon \rho} J_{\frac{n+|\gamma|}{2}-1}(\rho|\xi|) \rho^{\frac{n+|\gamma|}{2}} d \rho .
\end{gathered}
$$

Now, using the formula 2.12.8.4 from [22], p. 164 in the form

$$
\int_{0}^{\infty} x^{v+2} e^{-p x} J_{v}(c x) d x=\frac{2 p(2 c)^{v} \Gamma\left(v+\frac{3}{2}\right)}{\sqrt{\pi}\left(p^{2}+c^{2}\right)^{v+\frac{3}{2}}}, \quad \operatorname{Rev}>-1,
$$

we arrive at the representation

$$
\int_{0}^{\infty} e^{-\varepsilon \rho} J_{\frac{n+|\gamma|}{2}-1}(\rho|\xi|) \rho^{\frac{n+|\gamma|}{2}} d \rho=\frac{2 \varepsilon(2|\xi|)^{\frac{n+|\gamma|}{2}-1} \Gamma\left(\frac{n+|\gamma|+1}{2}\right)}{\sqrt{\pi}\left(\varepsilon^{2}+|\xi|^{2}\right)^{\frac{n+|\gamma|+1}{2}}} .
$$

Finally, we get the desired result:

$$
\begin{aligned}
\mathbf{F}_{\gamma}\left[e^{-\varepsilon|x|}\right](\xi) & =\frac{\prod_{i=1}^{n} \Gamma\left(\frac{\gamma_{i}+1}{2}\right)}{2^{\frac{n-|\gamma|}{2}}|\xi|^{\frac{n+|\gamma|}{2}}-1} \frac{2 \varepsilon(2|\xi|)^{\frac{n+|\gamma|}{2}}-1}{\sqrt{\pi}\left(\varepsilon^{2}+|\xi|^{2}\right)^{\frac{n+|\gamma|+\mid}{2}}}= \\
& =\frac{2^{|\gamma|} \mathcal{E} \prod_{i=1}^{n} \Gamma\left(\frac{\gamma_{i}+1}{2}\right) \Gamma\left(\frac{n+|\gamma|+1}{2}\right)}{\sqrt{\pi}\left(\varepsilon^{2}+|\xi|^{2}\right)^{\frac{n+|\gamma|+1}{2}}} .
\end{aligned}
$$

Now we deduce some properties of the general Poisson kernel needed for the further discussions. 
Lemma 2. The general Poisson kernel $P_{\gamma}(x, \varepsilon)$ possesses the following properties:

1. $\quad \mathbf{F}_{\gamma}\left[P_{\gamma}(x, \varepsilon)\right](\xi)=e^{-\varepsilon|\xi|,}$

2. $\int_{\mathbb{R}_{+}^{n}} P_{\gamma}(x, \varepsilon) x^{\gamma} d x=\int_{\mathbb{R}_{+}^{n}} P_{\gamma}(x, 1) x^{\gamma} d x=1$,

3. $P_{\gamma}(x, \varepsilon) \in L_{p}^{\gamma}, 1 \leq p \leq \infty$.

Proof. 1. This property immediately follows from Lemma 1.

2. By the variables substitution $x=\varepsilon y$, we first obtain

$$
\begin{gathered}
\int_{\mathbb{R}_{+}^{n}} P_{\gamma}(x, \varepsilon) x^{\gamma} d x=\frac{2^{n} \varepsilon \Gamma\left(\frac{n+|\gamma|+1}{2}\right)}{\sqrt{\pi} \prod_{j=1}^{n} \Gamma\left(\frac{\gamma_{j}+1}{2}\right)} \int_{\mathbb{R}_{+}^{n}} \frac{x^{\gamma} d x}{\left(\varepsilon^{2}+|x|^{2}\right)^{\frac{n+|\gamma|+1}{2}}}=\{x=\varepsilon y\}= \\
=\frac{2^{n} \Gamma\left(\frac{n+|\gamma|+1}{2}\right)}{\sqrt{\pi} \prod_{j=1}^{n} \Gamma\left(\frac{\gamma_{j}+1}{2}\right)} \int_{\mathbb{R}_{+}^{n}} \frac{y^{\gamma} d y}{\left(1+|y|^{2}\right)^{\frac{n+|\gamma|+1}{2}}}=\int_{\mathbb{R}_{+}^{n}} P_{\gamma}(x, 1) x^{\gamma} d x .
\end{gathered}
$$

Now we show that $\int_{\mathbb{R}_{+}^{n}} P_{\gamma}(x, 1) x^{\gamma} d x=1$. Indeed, first, we get the representation

$$
\int_{\mathbb{R}_{+}^{n}} \frac{y^{\gamma} d y}{\left(1+|y|^{2}\right)^{\frac{n+|\gamma|+1}{2}}}=\{y=\rho \sigma\}=\int_{0}^{\infty} \frac{\rho^{n+|\gamma|-1} d \rho}{\left(1+\rho^{2}\right)^{\frac{n+|\gamma|+1}{2}}} \int_{S_{1}^{+}(n)} \sigma^{\gamma} d S .
$$

To simplify the integral at the right-hand side of the last relation, we employ Equation (7) and obtain

$$
\begin{aligned}
\int_{\mathbb{R}_{+}^{n}} \frac{y^{\gamma} d y}{\left(1+|y|^{2}\right)^{\frac{n+|\gamma|+1}{2}}} & =\frac{\prod_{i=1}^{n} \Gamma\left(\frac{\gamma_{i}+1}{2}\right)}{2^{n-1} \Gamma\left(\frac{n+|\gamma|}{2}\right)} \int_{0}^{\infty} \frac{\rho^{n+|\gamma|-1} d \rho}{\left(1+\rho^{2}\right)^{\frac{n+|\gamma|+1}{2}}}=\left\{\rho^{2}=r\right\}= \\
& =\frac{\prod_{i=1}^{n} \Gamma\left(\frac{\gamma_{i}+1}{2}\right)}{2^{n} \Gamma\left(\frac{n+|\gamma|}{2}\right)} \int_{0}^{\infty} \frac{r^{\frac{n+|\gamma|}{2}}-1}{(1+r)^{\frac{n+|\gamma|+1}{2}}} d r .
\end{aligned}
$$

Now we use the formula 2.2.5.24 from [23], p. 239 in the form

$$
\int_{0}^{\infty} \frac{x^{\alpha-1}}{(x+z)^{\beta}} d x=z^{\alpha-\beta} B(\alpha, \beta-\alpha), \quad 0<\operatorname{Re} \alpha<\operatorname{Re} \beta,
$$

and obtain the relation

$$
2 \int_{0}^{\infty} \frac{\rho^{n+|\gamma|-1} d \rho}{\left(1+\rho^{2}\right)^{\frac{n+|\gamma|+1}{2}}}=\int_{0}^{\infty} \frac{r^{\frac{n+|\gamma|}{2}-1}}{(1+r)^{\frac{n+|\gamma|+1}{2}}} d r=\frac{\sqrt{\pi} \Gamma\left(\frac{n+|\gamma|}{2}\right)}{\Gamma\left(\frac{n+|\gamma|+1}{2}\right)} .
$$

Thus, the formula

$$
\int_{\mathbb{R}_{+}^{n}} \frac{y^{\gamma} d y}{\left(1+|y|^{2}\right)^{\frac{n+|\gamma|+1}{2}}}=\frac{\prod_{i=1}^{n} \Gamma\left(\frac{\gamma_{i}+1}{2}\right)}{2^{n} \Gamma\left(\frac{n+|\gamma|}{2}\right)} \frac{\sqrt{\pi} \Gamma\left(\frac{n+|\gamma|}{2}\right)}{\Gamma\left(\frac{n+|\gamma|+1}{2}\right)}=\frac{\sqrt{\pi} \prod_{i=1}^{n} \Gamma\left(\frac{\gamma_{i}+1}{2}\right)}{2^{n} \Gamma\left(\frac{n+|\gamma|+1}{2}\right)}
$$

holds valid. Finally, we get the desired property of the general Poisson kernel: 


$$
\int_{\mathbb{R}_{+}^{n}} P_{\gamma}(x, 1) x^{\gamma} d x=\frac{2^{n} \Gamma\left(\frac{n+|\gamma|+1}{2}\right)}{\sqrt{\pi} \prod_{j=1}^{n} \Gamma\left(\frac{\gamma_{j}+1}{2}\right)} \int_{\mathbb{R}_{+}^{n}} \frac{y^{\gamma} d y}{\left(1+|y|^{2}\right)^{\frac{n+|\gamma|+1}{2}}}=\frac{2^{n} \Gamma\left(\frac{n+|\gamma|+1}{2}\right)}{\sqrt{\pi} \prod_{j=1}^{n} \Gamma\left(\frac{\gamma_{j}+1}{2}\right)} \frac{\sqrt{\pi} \prod_{i=1}^{n} \Gamma\left(\frac{\gamma_{i}+1}{2}\right)}{2^{n} \Gamma\left(\frac{n+|\gamma|+1}{2}\right)}=1 .
$$

3. To show that $P_{\gamma}(x, \varepsilon) \in L_{p}^{\gamma}, 1 \leq p \leq \infty$, we start with the representation

$$
\begin{gathered}
\int_{\mathbb{R}_{+}^{n}} \frac{x^{\gamma} d x}{\left(\varepsilon^{2}+|x|^{2}\right)^{\frac{n+|\gamma|+1}{2}}}=\varepsilon^{(n+|\gamma|)(1-p)-p} \int_{\mathbb{R}_{+}^{n}} \frac{x^{\gamma} d x}{\left(|x|^{2}+1\right)^{\frac{n+|\gamma|+1}{2}}}= \\
=\{x=\rho \sigma,|x|=\rho\}=\varepsilon^{(n+|\gamma|)(1-p)-p} \int_{0}^{\infty} \frac{\rho^{n+|\gamma|-1} d \rho}{\left(\rho^{2}+1\right)^{p \frac{n+|\gamma|+1}{2}}} \int_{S_{1}^{+}(n)} \sigma^{\gamma} d S .
\end{gathered}
$$

For $1 \leq p<\infty$, we apply Equations (7) and (27) and obtain the following norm estimate:

$$
\begin{aligned}
\left\|P_{\gamma}(x, \varepsilon)\right\|_{p, \gamma} & =\left(\varepsilon^{(n+|\gamma|)(1-p)-p} \frac{\sqrt{\pi} \Gamma\left(\frac{n+|\gamma|}{2}\right)}{2 \Gamma\left(\frac{n+|\gamma|+1}{2}\right)} \frac{\prod_{i=1}^{n} \Gamma\left(\frac{\gamma_{i}+1}{2}\right)}{2^{n-1} \Gamma\left(\frac{n+|\gamma|}{2}\right)}\right)^{\frac{1}{p}}= \\
& =\left(\varepsilon^{(n+|\gamma|)(1-p)-p} \frac{\sqrt{\pi} \prod_{i=1}^{n} \Gamma\left(\frac{\gamma_{i}+1}{2}\right)}{2^{n} \Gamma\left(\frac{n+|\gamma|+1}{2}\right)}\right)^{\frac{1}{p}}<\infty
\end{aligned}
$$

In the case $p=+\infty$, the inequality $\left\|P_{\gamma}(x, \varepsilon)\right\|_{\infty, \gamma}<\infty$ is justified by passing to the limit $p \rightarrow \infty$ in the last formula and using the relation [16]

$$
\|f\|_{\infty, \gamma}=\lim _{p \rightarrow \infty}\|f\|_{p, \gamma}, \quad f \in L_{\infty}^{\gamma}
$$

\section{Inversion of the Bessel B-Potential by the Method of an Approximative Inverse Operator}

In this section, we derive an inverse operator to the generalized Bessel potential by applying the method of an approximative inverse operator.

The approximative inverse operator (AIO) method presented in [24] is one of the possible ways for the derivation of an inversion to certain convolution operators. The idea of the AIO method is that the inverse operator is constructed as the limit of a certain sequence of the approximative convolution operators with integrable kernels.

To apply this method for inversion of the Bessel B-potential, we first introduce the function

$$
\begin{gathered}
\omega_{\alpha, \gamma}(|x|)=\frac{2^{\frac{n-|\gamma|-\alpha}{2}+1}}{\Gamma\left(\frac{\alpha}{2}\right) \prod_{i=1}^{n} \Gamma\left(\frac{\gamma_{i}+1}{2}\right)}|x|^{\frac{n+|\gamma|-\alpha}{2}} K_{\frac{n+|\gamma|-\alpha}{2}}(|x|)= \\
=\frac{2^{n-\alpha+2}}{\Gamma\left(\frac{\alpha}{2}\right) \prod_{i=1}^{n} \Gamma\left(\frac{\gamma_{i}+1}{2}\right)} \int_{0}^{\infty} t^{\frac{n+|\gamma|-\alpha}{2}-1} e^{-t-\frac{|x|^{2}}{4 t}} d t .
\end{gathered}
$$

Then the generalized Bessel potential in Equation (1) can be represented as a convolution operator with respect to the generalized convolution defined by Equation (20):

$$
\mathbf{G}_{\gamma}^{\alpha} \varphi=\left(\frac{\omega_{\alpha, \gamma}(|x|)}{|x|^{n+|\gamma|-\alpha}} * \varphi\right)_{\gamma}, \quad \alpha>0 .
$$


Now we consider the convolution operators

$$
\left(\mathbf{G}_{\gamma, \varepsilon}^{\alpha}\right)^{-1} \varphi=\left(g_{\gamma, \varepsilon}^{\alpha} * \varphi\right)_{\gamma}
$$

with the kernel

$$
g_{\gamma, \varepsilon}^{\alpha}(x)=\left(\mathbf{F}_{\gamma}^{-1}\left(1+|\xi|^{2}\right)^{\alpha / 2} \cdot e^{-\varepsilon|\xi|}\right)(x) .
$$

To obtain a suitable integral representation of the kernel $g_{\gamma, \mathcal{\varepsilon}}^{\alpha}$, we change to the spherical coordinates $\xi=r \sigma, \sigma \in \mathbb{R}_{+}^{n}$ in the integral at the right-hand side of the previous relation and then apply Equation (8):

$$
\begin{gathered}
g_{\gamma, \varepsilon}^{\alpha}(x)=\frac{2^{n-|\gamma|}}{\prod_{j=1}^{n} \Gamma^{2}\left(\frac{\gamma_{j}+1}{2}\right)} \int_{\mathbb{R}_{+}^{n}}\left(1+|\xi|^{2}\right)^{\alpha / 2} \cdot e^{-\varepsilon|\xi|} \mathbf{j}_{\gamma}(x, \xi) \xi^{\gamma} d \xi= \\
=\frac{2^{n-|\gamma|}}{\prod_{j=1}^{n} \Gamma^{2}\left(\frac{\gamma_{j}+1}{2}\right)} \int_{0}^{\infty}\left(1+r^{2}\right)^{\alpha / 2} e^{-\varepsilon r} r^{n+|\gamma|-1} d r \int_{S_{1}^{+}(n)} \mathbf{j}_{\gamma}(x, r \sigma) \sigma^{\gamma} d S= \\
=\frac{2^{1-|\gamma|}}{\prod_{j=1}^{n} \Gamma\left(\frac{\gamma_{j}+1}{2}\right) \Gamma\left(\frac{n+|\gamma|}{2}\right)} \int_{0}^{\infty} j_{\frac{n+|\gamma|}{2}-1}(r|x|)\left(1+r^{2}\right)^{\alpha / 2} e^{-\varepsilon r} r^{n+|\gamma|-1} d r .
\end{gathered}
$$

Now we introduce the notion of a B-multiplier in $L_{p}^{\gamma}$. Let $M \in S_{e v}^{\prime}$. The weighted generalized function $M$ is called a B-multiplier in $L_{p}^{\gamma}$ if for $f \in S_{e v}$, the generalized convolution $\left(\mathbf{F}_{\gamma}^{-1} M * f\right)_{\gamma}$ belongs to the space $L_{p}^{\gamma}$ and the inequality

$$
\sup _{\|f\|_{p, \gamma}=1}\left\|\left(\mathbf{F}_{\gamma}^{-1} M * f\right)_{\gamma}\right\|_{p, \gamma}<+\infty
$$

holds true. The linear space of all B-multipliers is denoted by $M_{p, \gamma}=M_{p, \gamma}\left(\mathbb{R}_{+}^{n}\right)$. On the space $M_{p, \gamma}$, the left-hand side of the inequality in Equation (30) defines a norm that is used in the further discussion.

Lemma 3. Let $f \in S_{e v}$. The operator

$$
\left(\mathbf{G}_{\gamma, \varepsilon}^{\alpha}\right)^{-1} \varphi=\left(g_{\gamma, \varepsilon}^{\alpha} * \varphi\right)_{\gamma}=\int_{\mathbb{R}_{+}^{n}} g_{\gamma, \varepsilon}^{\alpha}(t)\left({ }^{\gamma} \mathbf{T}_{x}^{t} \varphi(x)\right) t^{\gamma} d t
$$

is bounded in $L_{p}^{\gamma}, 1<p<+\infty$.

Proof. Let $\varepsilon>0$ be a fixed number. The function $\left(1+|\xi|^{2}\right)^{\alpha / 2} \cdot e^{-\varepsilon|\xi|}$ is a B-multiplier for any $p, 1<p<+\infty$. Since the kernel function $g_{\gamma, \varepsilon}^{\alpha}(x)=\left(\mathbf{F}_{\gamma}^{-1}\left(1+|\xi|^{2}\right)^{\alpha / 2} \cdot e^{-\varepsilon|\xi|}\right)(x)$ is represented as an operator generated by the B-multiplier $\left(1+|\xi|^{2}\right)^{\alpha / 2} \cdot e^{-\varepsilon|\xi|}$ in $L_{p}^{\gamma}$, the inclusion $g_{\gamma, \varepsilon}^{\alpha}(x) \in L_{p}^{\gamma}$ holds valid.

Because the operator

$$
\left(\mathbf{G}_{\gamma, \varepsilon}^{\alpha}\right)^{-1} \varphi(x)=\left(\mathbf{F}_{\gamma}^{-1}\left[\left(1+|\xi|^{2}\right)^{\alpha / 2} \cdot e^{-\varepsilon|\xi|}\right](x) * \varphi(x)\right)_{\gamma}
$$

is a generalized convolution with a B-multiplier, its image also belongs to $L_{p}^{\gamma}$.

Lemma 4. Let $\varphi \in S_{e v}$. Then the formula

$$
\left(\left(\mathbf{G}_{\gamma}^{\alpha}\right)_{\varepsilon}^{-1} \mathbf{G}_{\gamma}^{\alpha} \varphi\right)(x)=\left(\mathbf{P}_{\gamma, \varepsilon} f\right)(x),
$$


holds valid, where $\left(\mathbf{P}_{\gamma, \varepsilon} f\right)(x)$ is the generalized convolution with the Poisson kernel

$$
\left(\mathbf{P}_{\gamma, \varepsilon} f\right)(x)=\left(f(x) * P_{\gamma}(x, \varepsilon)\right)_{\gamma} .
$$

Proof. By the convolution theorem, we get the relation

$$
\mathbf{F}_{\gamma}\left[\mathbf{G}_{\gamma}^{\alpha} \varphi\right](\xi)=\left(1+|\xi|^{2}\right)^{-\alpha / 2} \mathbf{F}_{\gamma} \varphi .
$$

Let us denote $\mathbf{G}_{\gamma}^{\alpha} \varphi$ by $\psi$. Then we have

$$
\begin{gathered}
\mathbf{F}_{\gamma}\left(\left(\mathbf{G}_{\gamma}^{\alpha}\right)_{\varepsilon}^{-1} \psi\right)(x)=\mathbf{F}_{\gamma}\left(\mathbf{F}_{\gamma}^{-1}\left[\left(1+|\xi|^{2}\right)^{\alpha / 2} \cdot e^{-\varepsilon|\xi|}\right](x) * \psi(x)\right)_{\gamma}= \\
=\left(1+|\xi|^{2}\right)^{\alpha / 2} \cdot e^{-\varepsilon|\xi|}(x) \cdot \mathbf{F}_{\gamma} \psi=\left(1+|\xi|^{2}\right)^{\alpha / 2} \cdot e^{-\varepsilon|\xi|}(x) \cdot\left(1+|\xi|^{2}\right)^{-\alpha / 2} \mathbf{F}_{\gamma} \varphi=e^{-\varepsilon|\xi|} \cdot \mathbf{F}_{\gamma} \varphi
\end{gathered}
$$

that leads to the representation

$$
\left(\left(\mathbf{G}_{\gamma}^{\alpha}\right)_{\varepsilon}^{-1} \mathbf{G}_{\gamma}^{\alpha} \varphi\right)(x)=\mathbf{F}_{\gamma}^{-1}\left(e^{-\varepsilon|\xi|} \cdot \mathbf{F}_{\gamma} \varphi\right)(x)=\left(\mathbf{F}_{\gamma}^{-1}\left(e^{-\varepsilon|\xi|}\right)(x) * \mathbf{F}_{\gamma} \varphi\right)_{\gamma}
$$

Applying Equation (26), we arrive at the relation

$$
\begin{gathered}
\left(\mathbf{F}_{\gamma}^{-1} e^{-\varepsilon|\xi|}\right)(x)=\frac{2^{n-|\gamma|}}{\prod_{j=1}^{n} \Gamma^{2}\left(\frac{\gamma_{j}+1}{2}\right)} \frac{2^{|\gamma|} \prod_{i=1}^{n} \Gamma\left(\frac{\gamma_{i}+1}{2}\right) \Gamma\left(\frac{n+|\gamma|+1}{2}\right)}{\sqrt{\pi}\left(\varepsilon^{2}+|x|^{2}\right)^{\frac{n+|\gamma|+1}{2}}}= \\
=\frac{2^{n} \Gamma\left(\frac{n+|\gamma|+1}{2}\right)}{\sqrt{\pi} \prod_{j=1}^{n} \Gamma\left(\frac{\gamma_{j}+1}{2}\right)} \varepsilon\left(\varepsilon^{2}+|x|^{2}\right)^{-\frac{n+|\gamma|+1}{2}}=P_{\gamma}(x, \varepsilon),
\end{gathered}
$$

where $P_{\gamma}(x, \varepsilon)$ is the generalized Poisson kernel in Equation (25). By Lemma 2, the inclusion $P_{\gamma}(x, \varepsilon) \in L_{p}^{\gamma}$ holds valid.

Theorem 1. Let $\varphi \in L_{p}^{\gamma}, 1 \leq p \leq+\infty$ and

$$
\left(\mathbf{G}_{\gamma}^{\alpha}\right)^{-1} \varphi(x)=\lim _{\varepsilon \rightarrow 0}^{L_{p}^{\gamma}}\left(\mathbf{G}_{\gamma, \varepsilon}^{\alpha}\right)^{-1} \varphi(x)=\left(\mathbf{F}_{\gamma}^{-1}\left[\left(1+|\xi|^{2}\right)^{\alpha / 2} \cdot e^{-\varepsilon|\xi|}\right](x) * \varphi(x)\right)_{\gamma}
$$

Then the relation

$$
\left(\mathbf{G}_{\gamma}^{\alpha}\right)^{-1} \mathbf{G}_{\gamma}^{\alpha} \varphi(x)=\varphi(x)
$$

holds valid, where the limit is understood in the sense of the norm in $L_{p}^{\gamma}$.

Proof. According to Lemma 4, we have to prove that

$$
\left\|\left(\mathbf{P}_{\gamma, \varepsilon} f\right)(x)-f(x)\right\|_{p, \gamma} \rightarrow 0 \quad \text { as } \quad \varepsilon \rightarrow 0 .
$$

Taking into account the 2nd property from Lemma 2, we get the representation

$$
\left(f(x) * P_{\gamma}(x, \varepsilon)\right)_{\gamma}-f(x)=\int_{\mathbb{R}_{+}^{n}}\left[{ }^{\gamma} \mathbf{T}_{x}^{y} f(x)-f(y)\right] P_{\gamma}(y, \varepsilon) y^{\gamma} d y .
$$

Applying the generalized Minkowski inequality [16] to the integral at the right-hand side of the last formula, we have

$$
\left\|\left(f(x) * P_{\gamma}(x, \varepsilon)\right)_{\gamma}-f(x)\right\|_{p, \gamma} \leq \int_{\mathbb{R}_{+}^{n}}\left(\int_{\mathbb{R}_{+}^{n}}\left[{ }^{\gamma} \mathbf{T}_{x}^{y} f(x)-f(x)\right]^{p} x^{\gamma} d x\right)^{\frac{1}{p}}\left|P_{\gamma}(y, \varepsilon)\right| y^{\gamma} d y=\{y=\varepsilon t\}
$$




$$
=\int_{\mathbb{R}_{+}^{n}}\left(\int_{\mathbb{R}_{+}^{n}}\left[{ }^{\gamma} \mathbf{T}_{x}^{\varepsilon t} f(x)-f(x)\right]^{p} x^{\gamma} d x\right)^{\frac{1}{p}}\left|P_{\gamma}(t, 1)\right| t^{\gamma} d t .
$$

Lemma 3.6 on p. 166 from [25] ensures that

$$
\left\|{ }^{\gamma} \mathbf{T}_{x}^{\varepsilon t} f(x)-f(x)\right\|_{p, \gamma} \leq c\|f(x)\|_{p, \gamma}
$$

for $f \in L_{p}^{\gamma}$. The Proposition 4.1, p. 182 from [26] and p. 50 from [27], leads to the formula

$$
\lim _{\varepsilon \rightarrow 0}\left(\int_{\mathbb{R}_{+}^{n}}\left[{ }^{\gamma} \mathbf{T}_{x}^{\varepsilon t} f(x)-f(x)\right]^{p} x^{\gamma} d x\right)^{\frac{1}{p}}=0 .
$$

By the Lebesgue majorized convergence theorem, the integral in Equation (33) tends to zero as $\varepsilon \rightarrow 0$ since the integrand is majorized by the integrable function $c|| f\left|\|_{p, \gamma}\right| P_{\gamma}(t, 1) \mid t^{\gamma}$.

\section{Inversion of the Bessel B-Potential by the Hadamard Finite Part Regularization Technique}

In this section, we construct another form of an inverse operator to the Bessel Bpotential using the concept of the "finite part" of a singular integral introduced by Hadamard.

Let a function $f=f(x)$ be integrable in a layer $\varepsilon<|x|<A$ for any $0<\varepsilon, \varepsilon<A<\infty$ and the representation

$$
\int_{\varepsilon<|x|<A} f(x) d x=\sum_{k=1}^{N} a_{k} \varepsilon^{-\lambda_{k}}+b \ln \frac{1}{\varepsilon}+J_{\varepsilon}
$$

hold valid, where $a_{k}, b$, and $\lambda_{k}$ are some constant positive numbers independent of $A$. If the $\operatorname{limit}_{\varepsilon \rightarrow 0} \lim _{\varepsilon}$ exists, then it is called the Hadamard finite part of the singular integral of the function $f$. The function $f=f(x)$ is said to possess the Hadamard property at the origin. The standard notation for the finite part of the Hadamard singular integral is as follows

$$
\text { p.f. } \int_{|x|<A} f(x) d x=\lim _{\varepsilon \rightarrow 0} J_{\varepsilon} .
$$

In the case $b=0$ in Equation (34), the function $f=f(x)$ is said to possess the nonlogarithmic-type Hadamard property at the origin.

If a function $f=f(x)$ is integrable on the set $\mathbb{R}_{+}^{n} \backslash\{|x|>\varepsilon\}$ and possesses the Hadamard property at the origin, then its finite part is defined as follows:

$$
p . f . \int_{\mathbb{R}_{+}^{n}} f(x) d x=p . f . \int_{|x|<A} f(x) d x+\int_{|x|>A} f(x) d x .
$$

We start by mentioning that the asymptotic properties of the modified Bessel function $K_{v}$ ensure that the kernel function $\omega_{-\alpha, \gamma}(|x|)$ exponentially decays at infinity (see Equation (16)) and goes to a constant at the origin (see Equation (15)):

$$
\lim _{|x| \rightarrow \infty} \omega_{-\alpha, \gamma}(|x|)=\frac{2^{\frac{n-|\gamma|+\alpha}{2}+1}}{\Gamma\left(-\frac{\alpha}{2}\right) \prod_{i=1}^{n} \Gamma\left(\frac{\gamma_{i}+1}{2}\right)} \lim _{|x| \rightarrow \infty}|x|^{\frac{n+|\gamma|+\alpha}{2}} K_{\frac{n+|\gamma|+\alpha}{2}}(|x|)=
$$




$$
\begin{gathered}
=\frac{2^{\frac{n-|\gamma|+\alpha}{2}+1}}{\Gamma\left(-\frac{\alpha}{2}\right) \prod_{i=1}^{n} \Gamma\left(\frac{\gamma_{i}+1}{2}\right)} \sqrt{\frac{\pi}{2}} \lim _{|x| \rightarrow \infty}|x|^{\frac{n+|\gamma|+\alpha}{2}-\frac{1}{2}} e^{-|x|}=0, \\
\lim _{|x| \rightarrow 0} \omega_{-\alpha, \gamma}(|x|)=\frac{2^{\frac{n-|\gamma|+\alpha}{2}+1}}{\Gamma\left(-\frac{\alpha}{2}\right) \prod_{i=1}^{n} \Gamma\left(\frac{\gamma_{i}+1}{2}\right)} \lim _{|x| \rightarrow 0}|x|^{\frac{n+|\gamma|+\alpha}{2}} K_{\frac{n+|\gamma|+\alpha}{2}}(|x|)= \\
=\frac{2^{\frac{n-|\gamma|+\alpha}{2}+1}}{\Gamma\left(-\frac{\alpha}{2}\right) \prod_{i=1}^{n} \Gamma\left(\frac{\gamma_{i}+1}{2}\right)} \lim _{|x| \rightarrow 0}|x|^{\frac{n+|\gamma|+\alpha}{2}} \frac{\Gamma\left(\frac{n+|\gamma|+\alpha}{2}\right)}{2^{1-\frac{n+|\gamma|+\alpha}{2}}}|x|^{\frac{n+|\gamma|+\alpha}{2}}=\frac{2^{n+\alpha} \Gamma\left(\frac{n+|\gamma|+\alpha}{2}\right)}{\Gamma\left(-\frac{\alpha}{2}\right) \prod_{i=1}^{n} \Gamma\left(\frac{\gamma_{i}+1}{2}\right)} .
\end{gathered}
$$

Therefore,

$$
\lim _{|x| \rightarrow \infty} \omega_{-\alpha, \gamma}(|x|)=0, \quad \lim _{|x| \rightarrow 0} \omega_{-\alpha, \gamma}(|x|)=\frac{2^{n+\alpha} \Gamma\left(\frac{n+|\gamma|+\alpha}{2}\right)}{\Gamma\left(-\frac{\alpha}{2}\right) \prod_{i=1}^{n} \Gamma\left(\frac{\gamma_{i}+1}{2}\right)} .
$$

Let as note that the function $\Gamma\left(-\frac{\alpha}{2}\right)$ in the denominator of $\omega_{-\alpha, \gamma}(0)$ has the simple poles at the points $\alpha=0,2,4,6, \ldots$, and therefore, the weighted generalized function $\frac{1}{|x|^{n+|\gamma|-\alpha}}$ has the simple poles at the same points.

As already mentioned in the previous section, the operator $\mathbf{G}_{\gamma}^{\alpha}$ can be represented in terms of the generalized convolution, as in Equation (20)

$$
\mathbf{G}_{\gamma}^{\alpha} \varphi=\left(\frac{\omega_{\alpha, \gamma}(|x|)}{|x|^{n+|\gamma|-\alpha}} * \varphi\right)_{\gamma} \quad \alpha>0
$$

Then we can apply the convolution theorem for the multidimensional Hankel transform (Equation (24)) and obtain the relation

$$
\mathbf{F}_{\gamma}\left[\mathbf{G}_{\gamma}^{\alpha} \varphi\right]=\mathbf{F}_{\gamma}\left[\frac{\omega_{\alpha, \gamma}(|x|)}{|x|^{n+|\gamma|-\alpha}}\right] \cdot \mathbf{F}_{\gamma}[\varphi] .
$$

Furthermore, the inclusion $\frac{\omega_{\alpha, \gamma}(|x|)}{|x|^{n+|\gamma|-\alpha}} \in L_{\gamma}^{1}\left(\mathbb{R}_{+}^{n}\right)$ and the formula

$$
\mathbf{F}_{\gamma}\left[\frac{\omega_{\alpha, \gamma}(|x|)}{|x|^{n+|\gamma|-\alpha}}\right](\xi)=\left(1+|\xi|^{2}\right)^{-\alpha / 2}
$$

hold valid, and we arrive at the representation

$$
\mathbf{F}_{\gamma}\left[\mathbf{G}_{\gamma}^{\alpha} \varphi\right]=\left(1+|\xi|^{2}\right)^{-\alpha / 2} \cdot \mathbf{F}_{\gamma}[\varphi] .
$$

Because of the semi-group property for the generalized Bessel potential [15], its inverse operator can be constructed in the form of a regularization of the generalized convolution

$$
\left(\mathbf{G}_{\gamma}^{\alpha}\right)^{-1} \varphi=\left(\frac{\omega_{-\alpha, \gamma}(|x|)}{|x|^{n+|\gamma|+\alpha}} * \varphi\right)_{\gamma}, \quad \alpha>0 .
$$

The inverse operator $\left(\mathbf{G}_{\gamma}^{\alpha}\right)^{-1}$ can be formally represented in terms of the multidimensional Hankel transform as follows:

$$
\mathbf{F}_{\gamma}\left[\left(\mathbf{G}_{\gamma}^{\alpha}\right)^{-1} \varphi\right]=\mathbf{F}_{\gamma}\left[\frac{\omega_{-\alpha, \gamma}(|x|)}{|x|^{n+|\gamma|+\alpha}}\right] \cdot \mathbf{F}_{\gamma}[\varphi], \quad \alpha>0 .
$$


However, Equation (37) shows that the integrand of the integral in Equation (39) has a singularity at the origin with the order greater than the dimension $n$ of $\mathbb{R}_{+}^{n}$, and therefore, the integral diverges. Thus, this integral cannot be used in its present form and should be suitably regularized. In what follows, we present some results regarding the regularization of this integral by employing the method of the Hadamard finite part of the singular integrals and construct the inverse operator $\left(\mathbf{G}_{\gamma}^{\alpha}\right)^{-1}$ in the following form

$$
\left(\mathbf{G}_{\gamma}^{\alpha}\right)^{-1} \varphi=p \cdot f \cdot\left(\frac{\omega_{-\alpha, \gamma}(|x|)}{|x|^{n+|\gamma|+\alpha}} * f\right)_{\gamma} .
$$

For further derivations, some advanced results regarding asymptotic behavior of the modified Bessel function $K_{v}$ are needed. It is known ([28], p. 273) that the inclusion $r^{v} K_{v}(r) \in C^{\infty}(0, \infty), v \in \mathbb{R}$ holds valid. Moreover, we have the estimates

$$
\left|\frac{d^{m}}{d r^{m}} r^{v} K_{v}(r)\right| \leq c \cdot r^{v-\frac{1}{2}} e^{-r}
$$

for large values of $r$ and the estimates

$$
\left|\frac{d^{m}}{d r^{m}} r^{v} K_{v}(r)\right| \leq c \begin{cases}r^{\theta} & \text { if } m<2 v, v>0 \\ r^{2 v-m} & \text { if } m>2 v\end{cases}
$$

for small values of $r$, where $\theta=1$ if $m$ is odd, and $\theta=0$ if $m$ is even, and $v$ is not an integer. If $v \in \mathbb{Z}$, then

$$
\left|\frac{d^{m}}{d r^{m}} r^{v} K_{v}(r)\right| \leq c \begin{cases}1 & \text { if } m<2 v, v>0 \\ r^{2 v-m} \ln \frac{1}{r} & \text { if } m>2 v .\end{cases}
$$

For $m<2 v$, the formula

$$
\frac{d^{m}}{d r^{m}} r^{v} K_{v}(r)=(-1)^{\frac{m}{2}} 2^{v-\frac{m}{2}-1}(m-1) ! ! \Gamma\left(v-\frac{m}{2}\right), \quad m=2,4,6, \ldots<2 v
$$

holds valid.

Equations (40)-(42) lead to the following statement:

Lemma 5. For $m<n+|\gamma|+\alpha$, the inclusion $\omega_{-\alpha, \gamma}(r) \in C^{m}([0, \infty])$ holds valid as well as the formulas

$$
\omega_{-\alpha, \gamma}^{(m)}(0)=0, \quad m=1,3,5, \ldots<n+|\gamma|+\alpha,
$$

$$
\omega_{-\alpha, \gamma}^{(m)}(0)=\frac{(-1)^{\frac{m}{2}} 2^{n+\alpha-\frac{m}{2}}(m-1) ! ! \Gamma\left(\frac{n+|\gamma|+\alpha}{2}-\frac{m}{2}\right)}{\Gamma\left(-\frac{\alpha}{2}\right) \prod_{i=1}^{n} \Gamma\left(\frac{\gamma_{i}+1}{2}\right)}, \quad m=2,4,6, \ldots<n+|\gamma|+\alpha .
$$

In the next theorem, a regularization of the generalized convolution $\left(\frac{\omega_{-\alpha, \gamma}(|x|)}{|x|^{n+|\gamma|+\alpha}} * \varphi\right)_{\gamma}$ is introduced using the appropriate segment of the Taylor-Delsarte series of the function $\varphi$. 
Theorem 2. Let $\varphi(x) \in S_{e v}\left(\mathbb{R}_{+}^{n}\right)$ and $\alpha>0$. Then the representation

$$
\begin{gathered}
p . f .\left(\frac{\omega_{-\alpha, \gamma}(|x|)}{|x|^{n+|\gamma|+\alpha}} * \varphi\right)_{\gamma}=\int_{\mathbb{R}_{+}^{n}} \frac{\gamma \mathbf{T}_{x}^{y} \varphi(x)-\left(P_{y}^{l-1} \varphi\right)(x)}{|y|^{n+|\gamma|+\alpha}} \omega_{-\alpha, \gamma}(|y|) y^{\gamma} d y+ \\
+\sum_{|2 m| \leq l-1} \zeta_{m}^{\gamma}\left(\mathcal{B}_{x}^{m} \varphi\right)(x) \frac{2^{2|m|} \prod_{i=1}^{n} \Gamma\left(\frac{\gamma_{i}+1}{2}+m_{i}\right) \Gamma\left(|m|-\frac{\alpha}{2}\right)}{\Gamma\left(-\frac{\alpha}{2}\right) \prod_{i=1}^{n} \Gamma\left(\frac{\gamma_{i}+1}{2}\right)}
\end{gathered}
$$

holds valid, where $\alpha<l \in \mathbb{N},\left(P_{y}^{l-1} \varphi\right)(x)=\sum_{|2 m| \leq l-1} \zeta_{m}^{\gamma}\left(\mathcal{B}_{x}^{m} \varphi\right)(x) y^{2 m}$ is a segment of the TaylorDelsarte series, $m=\left(m_{1}, \ldots, m_{n}\right)$ is the multi-index of non-negative integers, $y^{2 m}=y_{1}^{2 m_{1}} \ldots y_{n}^{2 m_{n}}$, $\left(\mathcal{B}_{x}^{m} \varphi\right)(x)=B_{\gamma_{1}}^{m_{1}} \ldots B_{\gamma_{n}}^{m_{n}} \varphi\left(x_{1}, \ldots, x_{n}\right), \zeta_{m}^{\gamma}=\frac{1}{2^{2|m|} m !} \prod_{i=1}^{n} \frac{\Gamma\left(\frac{\gamma_{i}+1}{2}\right)}{\Gamma\left(m_{i}+\frac{\gamma_{i}+1}{2}\right)}$, and $m !=m_{1} ! \cdot \ldots \cdot m_{n} !$

Proof. We start with the representation

$$
\begin{gathered}
p \cdot f \cdot\left(\frac{\omega_{-\alpha, \gamma}(|x|)}{|x|^{n+|\gamma|+\alpha}} * \varphi\right)_{\gamma}=p \cdot f \cdot \int_{\mathbb{R}_{+}^{n}} \frac{\omega_{-\alpha, \gamma}(|y|)^{\gamma} \mathbf{T}_{x}^{y} \varphi(x)}{|y|^{n+|\gamma|+\alpha}} y^{\gamma} d y= \\
=\int_{\mathbb{R}_{+}^{n}} \frac{\gamma \mathbf{T}_{x}^{y} \varphi(x)-\left(P_{y}^{l-1} \varphi\right)(x)}{|y|^{n+|\gamma|+\alpha}} \omega_{-\alpha, \gamma}(|y|) y^{\gamma} d y+p \cdot f \cdot \int_{\mathbb{R}_{+}^{n}} \frac{\left(P_{y}^{l-1} \varphi\right)(x)}{|y|^{n+|\gamma|+\alpha}} \omega_{-\alpha, \gamma}(|y|) y^{\gamma} d y,
\end{gathered}
$$

where

$$
\left(P_{y}^{l-1} \varphi\right)(x)=\sum_{|2 m| \leq l-1} \zeta_{m}^{\gamma}\left(\mathcal{B}_{x}^{m} \varphi\right)(x) y^{2 m}
$$

is a segment of the Taylor-Delsarte series, $l>\alpha$. Then we have the relation

$$
\begin{gathered}
p \cdot f \cdot \int_{\mathbb{R}_{+}^{n}} \frac{\left(P_{y}^{l-1} \varphi\right)(x)}{|y|^{n+|\gamma|+\alpha}} \omega_{-\alpha, \gamma}(|y|) y^{\gamma} d y \\
=\sum_{|2 m| \leq l-1} \zeta_{m}^{\gamma}\left(\mathcal{B}_{x}^{m} \varphi\right)(x) p \cdot f \cdot \int_{\mathbb{R}_{+}^{n}} \frac{y^{2 m}}{|y|^{n+|\gamma|+\alpha}} \omega_{-\alpha, \gamma}(|y|) y^{\gamma} d y .
\end{gathered}
$$

In order to derive a suitable representation for $p . f \cdot \int_{\mathbb{R}_{+}^{n}} \frac{y^{2 m}}{|y|^{n+|\gamma|+\alpha}} \omega_{-\alpha, \gamma}(|y|) y^{\gamma} d y$, let us consider an integral with an addition parameter $\beta$

$$
\mathcal{I}_{\alpha, \beta, \gamma}=\int_{\mathbb{R}_{+}^{n}} \frac{y^{2 m}}{|y|^{n+|\gamma|+\beta}} \omega_{-\alpha, \gamma}(|y|) y^{\gamma} d y, \quad \beta \in \mathbb{C},
$$

and suppose that $\alpha>0$ is fixed. tation

Passing to the spherical coordinates in the integral $\mathcal{I}_{\alpha, \beta, \gamma}$, we first obtain the represen-

$$
\mathcal{I}_{\alpha, \beta, \gamma}=\int_{\mathbb{R}_{+}^{n}} \frac{y^{2 m}}{|y|^{n+|\gamma|+\beta}} \omega_{-\alpha, \gamma}(|y|) y^{\gamma} d y=\{y=r \sigma\}=\int_{0}^{\infty} r^{2|m|-\beta-1} \omega_{-\alpha, \gamma}(r) d r \int_{S_{1}^{+}(n)} \sigma^{\gamma+2 m} d S .
$$


Using the formula 1.107, p. 49 from [16], we then get

$$
\int_{S_{1}^{+}(n)} \sigma^{2 m+\gamma} d S=\frac{\prod_{i=1}^{n} \Gamma\left(\frac{\gamma_{i}+1}{2}+m_{i}\right)}{2^{n-1} \Gamma\left(\frac{n+|\gamma|}{2}+|m|\right)}=I_{m}^{\gamma}
$$

and

$$
\begin{gathered}
\mathcal{I}_{\alpha, \beta, \gamma}=I_{m}^{\gamma} \int_{0}^{\infty} r^{2|m|-\beta-1} \omega_{-\alpha, \gamma}(r) d r= \\
=I_{m}^{\gamma} \frac{2^{\frac{n-|\gamma|+\alpha}{2}}+1}{\Gamma\left(-\frac{\alpha}{2}\right) \prod_{i=1}^{n} \Gamma\left(\frac{\gamma_{i}+1}{2}\right)} \int_{0}^{\infty} r^{2|m|-\beta-1+\frac{n+|\gamma|+\alpha}{2}} K_{\frac{n+|\gamma|+\alpha}{2}}(r) d r .
\end{gathered}
$$

The next step is applying the formula 2.16.2 from [22] in the form

$$
\int_{0}^{\infty} x^{a-1} K_{v}(c x) d x=2^{a-2} c^{-a} \Gamma\left(\frac{a+v}{2}\right) \Gamma\left(\frac{a-v}{2}\right), \quad \Re(a)>|\Re(v)|, \quad c>0 .
$$

In our case, the parameters are as follows: $a=2|m|-\beta+\frac{n+|\gamma|+\alpha}{2}, v=\frac{n+|\gamma|+\alpha}{2}>0$, and the convergence condition takes the form of $2|m|-\Re(\beta)+\frac{n+|\gamma|+\alpha}{2}>\frac{n+|\gamma|+\alpha}{2}$ or $\Re(\beta)<0$ since $|m|=0,1,2, \ldots$. Thus, we can calculate the integral $\mathcal{I}_{\alpha, \beta, \gamma}$ under the condition $\Re(\beta)<0$ :

$$
\mathcal{I}_{\alpha, \beta, \gamma}=I_{m}^{\gamma} \frac{2^{n+2|m|+\alpha-\beta-1}}{\Gamma\left(-\frac{\alpha}{2}\right) \prod_{i=1}^{n} \Gamma\left(\frac{\gamma_{i}+1}{2}\right)} \Gamma\left(|m|+\frac{n+|\gamma|+\alpha-\beta}{2}\right) \Gamma\left(|m|-\frac{\beta}{2}\right) .
$$

The formula above can be analytically extended with respect to $\beta$ to the half-plane $\Re(\beta)>0$. Setting $\beta=\alpha$ in this extended formula, we arrive at the representation

$$
p . f . \int_{\mathbb{R}_{+}^{n}} \frac{y^{2 m}}{|y|^{n+|\gamma|+\alpha}} \omega_{-\alpha, \gamma}(|y|) y^{\gamma} d y=I_{m}^{\gamma} \frac{2^{n+2|m|-1} \Gamma\left(|m|+\frac{n+|\gamma|}{2}\right) \Gamma\left(|m|-\frac{\alpha}{2}\right)}{\Gamma\left(-\frac{\alpha}{2}\right) \prod_{i=1}^{n} \Gamma\left(\frac{\gamma_{i}+1}{2}\right)} .
$$

Substituting the value of the integral $I_{m}^{\gamma}$ into the formula

$$
\begin{aligned}
& p . f .\left(\frac{\omega_{-\alpha, \gamma}(|x|)}{|x|^{n+|\gamma|+\alpha}} * \varphi\right)_{\gamma}=\int_{\mathbb{R}_{+}^{n}} \frac{{ }^{\gamma} \mathbf{T}_{x}^{y} \varphi(x)-\left(P_{y}^{l-1} \varphi\right)(x)}{|y|^{n+|\gamma|+\alpha}} \omega_{-\alpha, \gamma}(|y|) y^{\gamma} d y+ \\
& \quad+\sum_{|2 m| \leq l-1} \zeta_{m}^{\gamma}\left(\mathcal{B}_{x}^{m} \varphi\right)(x) I_{m}^{\gamma} \frac{2^{n+2|m|-1} \Gamma\left(|m|+\frac{n+|\gamma|}{2}\right) \Gamma\left(|m|-\frac{\alpha}{2}\right)}{\Gamma\left(-\frac{\alpha}{2}\right) \prod_{i=1}^{n} \Gamma\left(\frac{\gamma_{i}+1}{2}\right)}
\end{aligned}
$$

we arrive at the final result in Equation (45). 
Now we proceed with a construction of an inversion of the Bessel B-potential by the Hadamard finite part regularization technique. For $\varphi \in S_{e v}\left(\mathbb{R}_{+}^{n}\right)$ and $\alpha>0$, we define the operator

$$
\begin{gathered}
\left(I-\Delta_{\gamma}\right)^{\frac{\alpha}{2}} \varphi=\int_{\mathbb{R}_{+}^{n}} \frac{\gamma^{\gamma} \mathbf{T}_{x}^{y} \varphi(x)-\left(P_{y}^{l-1} \varphi\right)(x)}{|y|^{n+|\gamma|+\alpha}} \omega_{-\alpha, \gamma}(|y|) y^{\gamma} d y+ \\
+\sum_{|2 m| \leq l-1} \zeta_{m}^{\gamma}\left(\mathcal{B}_{x}^{m} \varphi\right)(x) \frac{2^{2|m|} \prod_{i=1}^{n} \Gamma\left(\frac{\gamma_{i}+1}{2}+m_{i}\right) \Gamma\left(|m|-\frac{\alpha}{2}\right)}{\Gamma\left(-\frac{\alpha}{2}\right) \prod_{i=1}^{n} \Gamma\left(\frac{\gamma_{i}+1}{2}\right)}
\end{gathered}
$$

that turns to be inverse to the Bessel B-potential $\mathbf{G}_{\gamma}^{\alpha} \varphi$.

Theorem 3. On the space $S_{e v}$, the operator $\left(I-\Delta_{\gamma}\right)^{\frac{\alpha}{2}}, \Re(\alpha)>0$ defined by Equation (46) is the right-and left-inverse to the Bessel B-potential, i.e., the formulas

$$
\left(I-\Delta_{\gamma}\right)^{\frac{\alpha}{2}} \mathbf{G}_{\gamma}^{\alpha} \varphi=\mathbf{G}_{\gamma}^{\alpha}\left(I-\Delta_{\gamma}\right)^{\frac{\alpha}{2}} \varphi=\varphi
$$

hold valid for any $\varphi \in S_{e v}$.

Proof. The main idea of the proof is first to consider the generalized convolution of the weighted functional $\mathbf{F}_{\gamma}^{-1}\left(1+|x|^{2}\right)^{\alpha / 2}$ with a function $f$ from $S_{e v}$. Then an analytic continuation of the weighted functional $\mathbf{F}_{\gamma}^{-1}\left(1+|x|^{2}\right)^{\alpha / 2}$ with respect to $\alpha$ is constructed. It turns out that the generalized convolution of this analytic continuation with a function $f$ from $S_{e v}$ coincides with the representation in Equation (46). The final part of the proof involves the application of the inverse multidimensional Hankel transform.

Now we consider the steps mentioned above in detail and start by considering the inverse Hankel integral transform $\mathbf{F}_{\gamma}^{-1}\left(1+|x|^{2}\right)^{\alpha / 2}$ in the sense of the weighted distributions from the space $S_{e v}^{\prime}$. On the space $S_{e v}$, the operator inverse to $\mathbf{G}_{\gamma}^{\alpha}$ has the form

$$
\left(\mathbf{G}_{\gamma}^{\alpha}\right)^{-1} \varphi=\left(\mathbf{F}_{\gamma}^{-1}\left(1+|x|^{2}\right)^{\alpha / 2} * \varphi\right)_{\gamma}=\left(\mathbf{F}_{\gamma}^{-1}\left(1+|x|^{2}\right)^{\alpha / 2},{ }^{\gamma} \mathbf{T}_{x}^{y} \varphi\right)_{\gamma}, \varphi \in S_{e v} .
$$

For $\varphi \in S_{e v}$, the generalized convolution from the right-hand side of the last formula is analytical with respect to the parameter $\alpha$ on the whole complex plane.

Let us introduce a parameter $\beta \in \mathbb{C}$ that is not an integer number. It is known (see [21]) that the inclusion $f \in S_{e v}$ holds valid for $f=\gamma^{\gamma} \mathbf{T}_{x}^{y} \varphi$ and $\varphi \in S_{e v}$. Thus, for

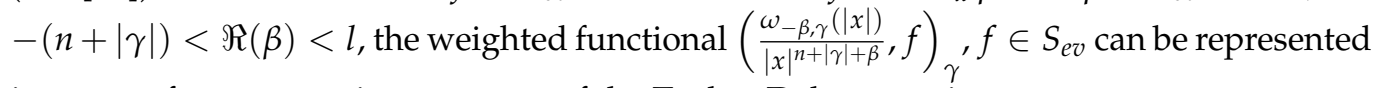
in terms of an appropriate segment of the Taylor-Delsarte series:

$$
\left(\frac{\omega_{-\beta, \gamma}(|x|)}{|x|^{n+|\gamma|+\beta}}, f\right)_{\gamma}=
$$

$$
=\int_{\mathbb{R}_{+}^{n}} \frac{f(y)-\left(P_{y}^{l-1} f\right)(0)}{|y|^{n+|\gamma|+\beta}} \omega_{-\beta, \gamma}(|y|) y^{\gamma} d y+\sum_{|2 m| \leq l-1} \zeta_{m}^{\gamma}\left(\mathcal{B}_{x}^{m} f\right)(0) I_{m}^{\gamma} \frac{2^{n+2|m|-1} \Gamma\left(|m|+\frac{n+|\gamma|}{2}\right) \Gamma\left(|m|-\frac{\beta}{2}\right)}{\Gamma\left(-\frac{\beta}{2}\right) \prod_{i=1}^{n} \Gamma\left(\frac{\gamma_{i}+1}{2}\right)},
$$

where $\left(P_{y}^{l-1} f\right)(0)=\sum_{|2 m| \leq l-1} \zeta_{m}^{\gamma}\left(\mathcal{B}_{x}^{m} f\right)(0) y^{2 m}$. The integral at the right-hand side of Equation (48) is analytical with respect to the parameter $\beta$ in the domain $-(n+|\gamma|)<\Re(\beta)<l$ since the expression $\left(f(y)-\left(P_{y}^{l-1} f\right)(0)\right)$ compensates the singularity of the denominator at the origin as in the case of the B-hyper-singular integral with a homogeneous characteristic [29]. Therefore, the weighed functional in Equation (48) is analytic with respect to $\beta$ in the domain $-(n+|\gamma|)<\Re(\beta)<l$. Thus, under the condition $-(n+|\gamma|)<\Re(\beta)<0$, we arrive at the representation

$$
\left(\frac{\omega_{-\beta, \gamma}(|x|)}{|x|^{n+|\gamma|+\beta}}, f\right)_{\gamma}=\int_{\mathbb{R}_{+}^{n}} \frac{\omega_{-\beta, \gamma}(|y|)}{|y|^{n+|\gamma|+\beta}} f(y) y^{\gamma} d y=\left(\mathbf{F}_{\gamma}^{-1}\left(1+|x|^{2}\right)^{\beta / 2}, f\right)_{\gamma}, \quad f \in S_{e v} .
$$


In its turn, the weighed functional on the right-hand side of Equation (49) is analytic with respect to $\beta$ in the whole complex plane because it can be represented in the form

$$
\left(\mathbf{F}_{\gamma}^{-1}\left(1+|x|^{2}\right)^{\beta / 2}, f\right)_{\gamma}=\frac{2^{n-|\gamma|}}{\prod_{j=1}^{n} \Gamma^{2}\left(\frac{\gamma_{j}+1}{2}\right)}\left(\left(1+|x|^{2}\right)^{\beta / 2}, \mathbf{F}_{\gamma} f\right)_{\gamma}
$$

and because all weighted functions $\left(1+|x|^{2}\right)^{\beta / 2}$ are analytic with respect to $\beta$ (see Chapter 4 in [16]). Therefore, the representation in Equation (49) also keeps its validity in the case $\beta=\alpha, 0<\Re(\alpha)<l$. Using the relation $f={ }^{\gamma} \mathbf{T}_{x}^{y} \varphi$, we rewrite the representation from Equation (48) for the function $\varphi$ as follows:

$$
\left(\mathbf{G}_{\gamma}^{\alpha}\right)^{-1} \varphi=\left(\mathbf{F}_{\gamma}^{-1}\left(1+|x|^{2}\right)^{\alpha / 2} * \varphi\right)_{\gamma}=\left(I-\Delta_{\gamma}\right)^{\frac{\alpha}{2}} \varphi,
$$

where $\left(I-\Delta_{\gamma}\right)^{\frac{\alpha}{2}}$ is defined as in Equation (46). Equations (47) now follow from their representations as the images of the Hankel integral transform. To derive the first formula, we first get the representation

$\mathbf{F}_{\gamma}\left(I-\Delta_{\gamma}\right)^{\frac{\alpha}{2}} \mathbf{G}_{\gamma}^{\alpha} \varphi=\mathbf{F}_{\gamma}\left(\mathbf{F}_{\gamma}^{-1}\left(1+|x|^{2}\right)^{\alpha / 2} * \mathbf{G}_{\gamma}^{\alpha} \varphi\right)_{\gamma}=\left(1+|x|^{2}\right)^{\alpha / 2} \cdot \mathbf{F}_{\gamma} \mathbf{G}_{\gamma}^{\alpha} \varphi=\left(1+|x|^{2}\right)^{\alpha / 2} \cdot\left(1+|\xi|^{2}\right)^{-\alpha / 2} \mathbf{F}_{\gamma} \varphi=\mathbf{F}_{\gamma} \varphi$.

Applying the inverse Hankel transform to the last formula, we immediately arrive at the desired relation $\left(I-\Delta_{\gamma}\right)^{\frac{\alpha}{2}} \mathbf{G}_{\gamma}^{\alpha} \varphi=\varphi$. The second formula from Equation (47), i.e., $\mathbf{G}_{\gamma}^{\alpha}\left(I-\Delta_{\gamma}\right)^{\frac{\alpha}{2}} \varphi=\varphi$ is proved by employing the same arguments.

Finally, let us consider a particular case of the inversion of the Bessel B-potential in the form of Equation (46) under the condition $0<\alpha<1$. In this case, for $l=1$, we have the formula $\left(P_{y}^{0} f\right)(x)=f(x)$ and the inverse operator in Equation (46) takes a simple form

$$
\left(I-\Delta_{\gamma}\right)^{\frac{\alpha}{2}} \varphi=\int_{\mathbb{R}_{+}^{n}} \frac{\gamma \mathbf{T}_{x}^{y} f(x)-f(x)}{|y|^{n+|\gamma|+\alpha}} \omega_{-\alpha, \gamma}(|y|) y^{\gamma} d y+f(x) .
$$

\section{Conclusions}

In this paper, we considered a convolution-type operator called the generalized Bessel potential. This is a very important object in the potential theory with several applications, including analytical treatment of the non-homogeneous equations of potential type, theory of functional spaces, and functional completion. Thus, a problem of the derivation of its inversion is worth investigation. In this paper, we presented two different forms of the operators inverse to the generalized Bessel potential. The first form was derived in terms of an approximative inverse operator using the method of an improving multiplier. The second form employs the regularization technique for the divergent integrals in terms of the appropriate segments of the Taylor-Delsarte series. It is worth mentioning that both inversions can be interpreted as new fractional differentiation operators of arbitrary positive order. Further research problems in this direction include detailed investigation of these new operators on different spaces of functions as well as the analysis of the partial differential equations of fractional order with the fractional derivatives in form of the inversions of the generalized Bessel potential.

Author Contributions: Conceptualization: Y.L. and E.S.; methodology: Y.L. and E.S.; formal analysis: A.D. and Y.L.; investigation: Y.L., A.D., and E.S.; resources: Y.L. and E.S.; writing-original draft preparation: Y.L., A.D., and E.S.; writing-review and editing: Y.L.; visualization: Y.L., A.D., and E.S.; supervision: Y.L.; project administration: Y.L. All authors have read and agreed to the published version of the manuscript.

Funding: This research received no external funding.

Institutional Review Board Statement: Not applicable. 
Informed Consent Statement: Not applicable.

Data Availability Statement: Not applicable.

Conflicts of Interest: The authors declare no conflicts of interest.

\section{Notations}

The following notations are used in this paper:

$\begin{array}{lll}\mathbf{G}^{\alpha} & \text { the generalized Bessel potential } & \text { Equation (1) } \\ G_{\alpha}^{\gamma} & \text { the generalized Bessel potential kernel } & \text { Equation (2) } \\ \Delta_{\gamma} & \text { the Laplace-Bessel operator } & \text { Equation (3) } \\ B_{\gamma} & \text { the Bessel operator } & \text { Equation (4) } \\ \mathbf{j}_{\gamma} & \text { the multidimensional normalized Bessel function of the first kind } & \text { Equation (9) } \\ j_{\nu} & \text { the normalized Bessel function of the first kind } & \text { Equation (10) } \\ { }_{\gamma} T_{x}^{y} & \text { the one-dimensional generalised translation } & \text { Equation (17) } \\ { }^{\gamma} \mathbf{T}_{x}^{y} & \text { the multidimensional generalized translation } & \text { Equation (19) } \\ (\cdot * \cdot)_{\gamma} & \text { the generalized convolution } & \text { Equation (20) } \\ \mathbf{F}_{\gamma} & \text { the multidimensional Hankel transform } & \text { Equation (23) } \\ P_{\gamma} & \text { the general Poisson kernel } & \text { Equation (25) } \\ g_{\gamma, \varepsilon}^{\alpha} & \text { the regularisation kernel } & \text { Equation (29) } \\ \mathbf{P}_{\gamma, \varepsilon} & \text { the generalized convolution with the Poisson kernel } & \text { Equation (31) } \\ p \cdot f . \int & \text { the finite part of the Hadamard singular integral } & \text { Equation (35) }\end{array}$

\section{References}

1. Aronszajn, N.; Smith, K.T. Theory of Bessel potentials. I. Ibid. 1961, 11, 365-475. [CrossRef]

2. Calderon, A.P. Lebesgue spaces of differentiable functions and distributions. In Proceedings of the Symposia in Pure Mathematics 4, Berkeley, CA, USA, 21-22 April 1960; Volume 4, pp. 33-50.

3. Stein, E.M. The characterization of functions arising as potentials. I. Bull. Amer. Math. Soc. 1961, 67, 102-104. [CrossRef]

4. Lizorkin, P.I. Characterization of the spaces $L_{p}^{r}\left(R_{n}\right)$ in terms of difference singular integrals. Russian Mat. Sb. 1970, 81, 79-91.

5. $\quad$ Nogin, V.A. Inversion of Bessel potentials. Differ. Equat. 1982, 18, 1407-1411.

6. Nogin, V.A. Inversion of Bessel potentials by means of hypersingular integrals. Soviet Math. 1985, $29,73-83$.

7. Guliev, V.S.; Safarov, Z.V. $B_{k, n}$-Bessel potentials and certain imbedding theorems in $B_{k, n}$-Sobolev-Liouville spaces. Proc. Inst. Math. Mech. Natl. Acad. Sci. Azerb. 2001, 15, 68-80.

8. Guliev, V.S.; Serbetci, A.; Akbulut, A.; Mammadov, Y.Y. Nikolskii-Besov and Lizorkin-Triebel spaces constructed on the base of the multidimensional Fourier-Bessel transform. Eurasian Math. J. 2011, 2, 42-66.

9. Gol'dman, M.L. Integral properties of generalized Bessel potentials. DAN 2007, 414, 159-164. [CrossRef]

10. Gol'dman, M.L. Rearrangement-invriant spans for generalized Bessel and Riesz Potentials. DAN 2008, 423, 14-18.

11. Gol'dman, M.L. The cone of rearrangements for generalized Bessel Potentials. Tr. Mat. Inst. Steklova 2008, 260, 151-163. [CrossRef]

12. Aliev, I.A.; Eryigit, M. Inversion of Bessel potentials with the aid of weighted wavelet transforms. Math. Nachr. 2002, 242, 27-37. [CrossRef]

13. Aliev, I.A.; Eryigit, M. On a rate of convergence of truncated hypersingular integrals associated to Riesz and Bessel potentials. J. Math. Anal. Appl. 2013, 406, 352-359. [CrossRef]

14. Aliev, I.A.; Gadjiev, A.D.; Aral, A. On approximation properties of a family of linear operators at critical value of parameter. J. Approx. Theory 2006, 138, 242-253. [CrossRef]

15. Shishkina, E.; Ekincioğlu, I.; Keskin, C. Generalized Bessel potential and its application to non-homogeneous singular screened Poisson equation. Integral Transform. Spec. Funct. 2020, 1-16. [CrossRef]

16. Shishkina, E.L.; Sitnik, S.M. Transmutations, Singular and Fractional Differential Equations with Applications to Mathematical Physics; Elsevier: Amsterdam, The Netherlands, 2020.

17. Kipriyanov, I.A. Singular Elliptic Boundary Value Problems; Nauka: Moscow, Russia, 1997.

18. Erdelyi, A.; Magnus, W.; Oberhettinger, F.; Tricomi, F.G. Higher Transcendental Functions; McGraw-Hill Book Co.: New York, NY, USA, 1953; Volume 2.

19. Watson, G.N. A Treatise on the Theory of Bessel Functions; University Press: Cambridge, UK, 1922.

20. Abramowitz, M.; Stegun, I.A. Handbook of Mathematical Functions with Formulas, Graphs and Mathematical Tables; Dover Publ., Inc.: New York, NY, USA, 1972.

21. Levitan, B.M. Expansion in Fourier Series and Integrals with Bessel Functions. Uspekhi Mat. Nauk 1951, 6, $102-143$.

22. Prudnikov, A.P.; Brychkov, Y.A.; Marichev, O.I. Integrals and Series, Vol. 2, Special Functions; Gordon \& Breach Sci. Publ.: New York, NY, USA, 1990. 
23. Prudnikov, A.P.; Brychkov, Y.A.; Marichev, O.I. Integrals and Series, Vol. 1, Elementary Functions; Gordon \& Breach Sci. Publ.: New York, NY, USA, 1992.

24. Samko, S.G. A new approach to the inversion of the Riesz potential operator. Fract. Calc. Appl. Anal. 1998, 1, 225-245.

25. Platonov, S.S. Bessel generalized translations and some problems of approximation theory for functions on the half-line. Sibirsk. Mat. Zh. 2009, 50, 154-174. [CrossRef]

26. Platonov, S.S. Bessel harmonic analysis and approximation of functions on a semiaxis. Izv. RAN. Ser. Mat. 2007, 71, 149-196.

27. Platonov, S.S. Generalized Bessel translations and some inverse theorems of the theory of approximation of functions on the half-line. Tr. PetrGU. Ser. Mat. 2007, 14, 44-57.

28. Samko, S.G. Hypersingular Integrals and Their Applications; Taylor \& Francis: London, UK, 2002.

29. Lyakhov, L.N.; Shishkina, E.L. General B-Hypersingular Integrals with Homogeneous Characteristic. DAN $2007,75,39-43$. [CrossRef] 